\title{
The Strategic Imperative For An Integrated Enterprise
}

\author{
Jian Guan, (Email: jeff.guan@louisville.edu), University of Louisville
}

Robert M. Barker, (Email: rmbark01@louisville.edu), University of Louisville

\begin{abstract}
Most large organizations today have invested heavily in business software applications over the past few decades. These applications support key elements of sales, manufacturing, customer service, financial operations and have reaped tremendous rewards in productivity gains and competitive advantage. However, these individual applications were often not built or purchased with interoperability in mind. Rather, they evolved over time as a result of the latest technological innovation or hasty business need, leading to extremely complex, incompatible systems. As a result in many large organizations today the IT infrastructure often consists of many islands of automation, characterized by heterogeneous computing platforms, various proprietary information formats and diverse programming models. Against this backdrop competitive pressures are forcing large organizations to improve efficiency through integrating key business operations. E-commerce initiatives are also calling for a more integrated enterprise. This paper first examines the problem of poorly integrated business applications and the inadequacy of the traditional point-to-point integration approach. The paper then presents today's enterprise application technology and discusses the advantages of enterprise application integration and e-commerce or B2B application integration.
\end{abstract}

\section{Introduction}

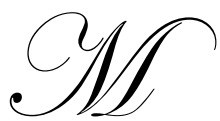

ost large organizations today have invested heavily in business software applications over the past few decades. These applications support key elements of sales, manufacturing, customer service, financial operations and have reaped tremendous rewards in productivity gains and competitive advantage. However, these individual applications were often not built or purchased with interoperability in mind. Rather, they evolved over time as a result of the latest technological innovation or hasty business need, leading to extremely complex, incompatible systems (Linthicum, 2000;). As a result in many large organizations today the IT infrastructure often consists of many islands of automation, characterized by heterogeneous computing platforms, various proprietary information formats and diverse programming models. Against this backdrop competitive pressures are forcing large organizations to improve efficiency through integrating key business operations. E-commerce initiatives are also calling for a more integrated enterprise.

This paper first examines the problem of poorly integrated business applications and the inadequacy of the traditional point-to-point integration approach. The paper then presents today's enterprise application technology and discusses the advantages of enterprise application integration and e-commerce or B2B application integration.

\section{The Application Integration Problem}

Many businesses have been creating islands of automation through generations of technology development and acquisition and are faced today with the daunting task of integrating these isolated applications (Leonard, 2000; Fulcher, 2000; Grimson, 2000; Yang et al, 2000;)(see Figure 1). According to one study up to $35 \%$ to $40 \%$ of development time is devoted to creating interfaces and points of integration for applications and data sources Readers with comments or questions are encouraged to contact the authors via email. 
Figure 1 Stovepipes

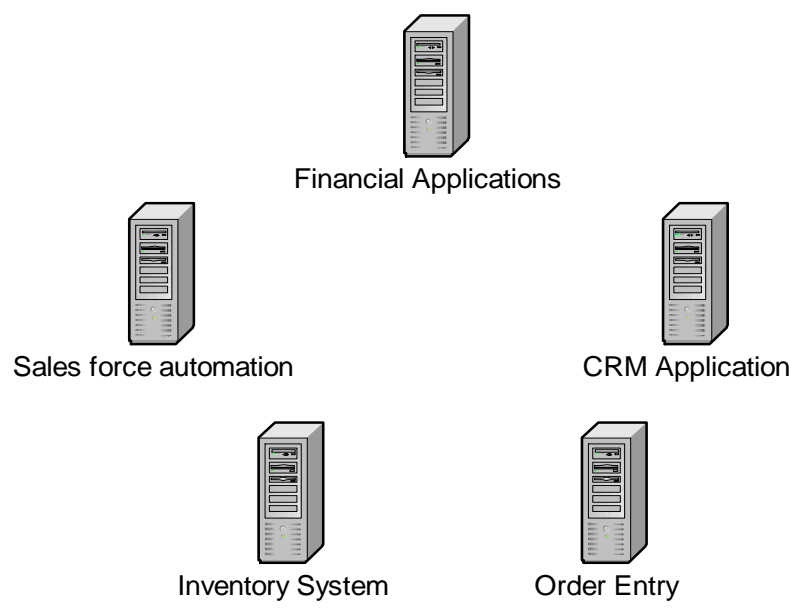

(Linthicum, 2000; Hey, 2000). Lou Gertner, CEO of IBM, said recently that the critical software in the networked world is no longer the operating system, it is the open middleware that ties together all the servers, all the applications, and all the access devices (McDougall, 2001). Though many of the applications found in companies today do a stellar job of coalescing a business process, they have no inherent capability to integrate with each other. In other words a basic enterprise financial system may enable transparency among accounts payable/receivable, general ledger, purchase-to-pay, fixed assets, and general accounting, but possesses no intrinsic connection to supply chain and/or customer relationship management systems.

A poorly integrated enterprise suffers from a variety of problems:

- Poor organizational flexibility. Adapting to changes in the business environment often means costly and time-consuming modifications to existing IT infrastructure, especially if the IT infrastructure is fragmented.

- Customer fragmentation. Customer information is scattered throughout many different systems. This directly leads to problems with customer support, customer retention, and loss of opportunities for cross selling.

- $\quad$ Business process fragmentation.

- $\quad$ Slower time to market.

- Inflated IT budget due to high application integration cost.

Two major factors have contributed to this common problem of islands of automation. First, the best of breed approach in software acquisition has been popular for some time (reference). This approach, however, has often led to a situation where there is a separate system for ordering, account management, billing, inventory, etc. The result is a bunch of stovepipe systems that either do not communicate or do not communicate well. Poor communication between these vital systems often leads to extremely complex business processes. Second, legacy systems also contribute to the complexity of application integration. Many of today's businesses still have to rely on these legacy systems for vital parts of their operations and replacing them with a newer system is often too expensive. Data, information, and business rules are often hard-coded into the legacy systems. Changing the legacy systems to adapt to changing business needs and changing IT infrastructure often requires major changes to the software. Such major modifications are either impossible, too difficult, or too time-consuming.

Traditionally, many companies have used point-to-point connections when it comes to integrating applications. In a point-to-point connection a custom interface is built between two applications. But custom connectivity or interface is expensive: the cost to custom-connect a pair of major applications runs as high as $\$ 10$ million (Brakeley, 2000). In addition as the number of applications increases, the number of point-to-point connections can rise exponentially. Figure 2 shows an example of five applications connected point-to-point. In this case, for five applications that both send and receive data, ten custom interfaces would be required. Each interface is costly to develop and requires cooperation among vendors. Moreover, if application requirements change or application is replaced, new interfaces must be developed 
Figure 2 Point to Point Connectivity

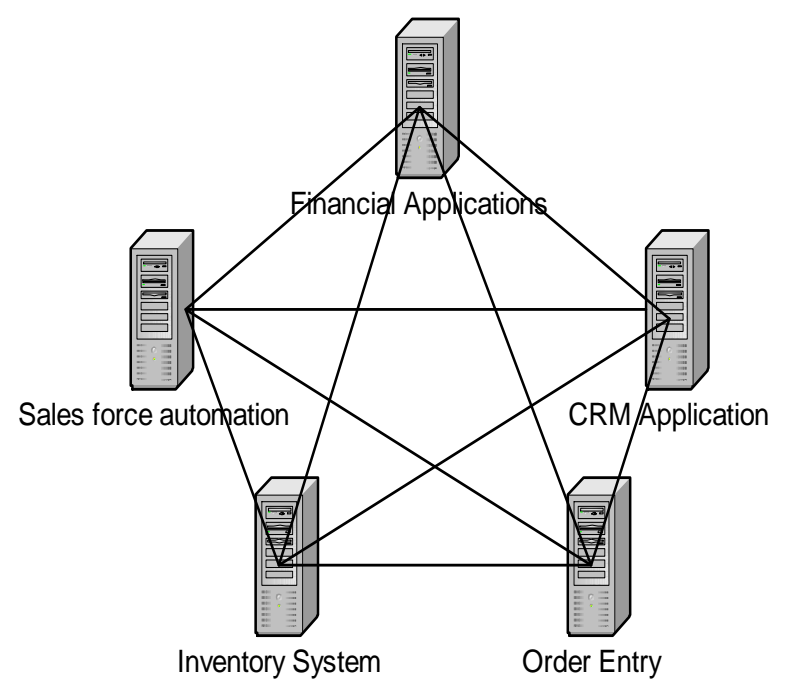

One of the main objectives of enterprise resource planning (ERP) systems has been to establish one database and working around a common workflow model for different functional areas of the same enterprise. While some companies have enjoyed significant gains in integrating business processes across organizational functions and locations, others have failed in their ERP efforts (Soh et al.). A common problem has been the issue of misfits or gaps between the functionality offered by the package and that required by the adopting organization (reference). Demands for specialized data, variances in systems among operating partners and the sheer diversity in available applications have made it virtually impossible for any company to manage all its business processes with one software package (Hey, 2000). New Internet applications based on Java, XML further complicate integration. The problem is exacerbated because ERP implementation is very complex, very expensive, and often takes a long time to implement. Even in successful implementations of ERP companies still have to tie ERP systems to internal and external applications.

\section{Enterprise Application Integration Technology}

The problems of a disconnected enterprise and the inadequacy of point-to-point approach to integration have prompted the emergence of a new breed of integration technology commonly referred to as enterprise application integration (EAI) technology. EAI software automates many key integration functions ranging from interfacing with multiple applications, to translating data structures, to intelligently routing messages, to creating vital business processes. This section briefly discusses the highlights or key features of the EAI technology.

Table 1 Key Features of EAI Technology Today

\begin{tabular}{|c|}
\hline Hub and spoke architecture \\
\hline Message brokering \\
\hline $\begin{array}{c}\text { Loose coupling of applications through use of adap- } \\
\text { ters }\end{array}$ \\
\hline Support for business process automation \\
\hline Support for B2B integration \\
\hline
\end{tabular}

cation and the other applications are not affected.
At the heart of today's EAI technology is the hub-and-spoke approach to integration (see Figure 3). This architecture eliminates the need for point-to-point connections. Each application connects to the hub and this allows data translation and routing functions to occur in a single location. This solution is more cost effective to implement than the point-to-point approach. If an application is upgraded or replaced, interface changes are handled at the application integration level for that appli-

In the center of such a hub-and-spoke integration architecture sits the message broker. A message broker is a software system based on asynchronous, store-and-forward messaging. It manages interactions between applications and other information resources. An application publishes a message to the message broker and other applications subscribe to the message. Such an architecture allows true any-to-any and many-to-many connectivity. The message broker mediates the interactions between the applications thus the applications (either as publishers of information or subscribers of information) can remain truly anonymous. 


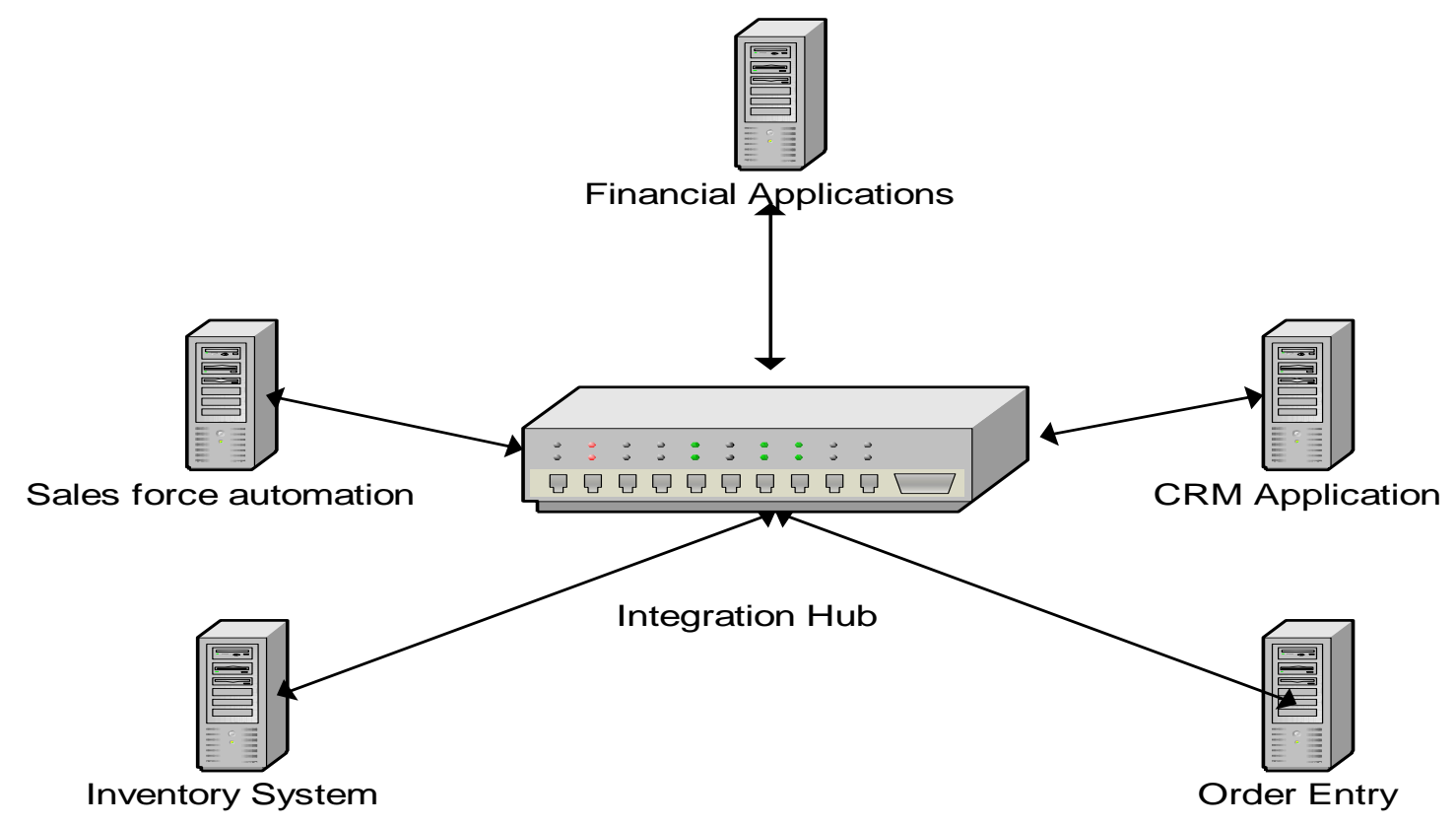

Adapters are layers between the message broker and the source or target application. It maps the differences between two distinct interfaces - the message broker interface and the native interface of the source or target application - and that hide the complexities of that interface.

Support for business process automation is another key feature of EAI technology. Most business processes have already been automated but they tend to be loosely coupled and exist in different applications. Process automation is the passing of information from participating application to participating application and the execution of appropriate rules in order to achieve a certain business objective. The establishment of an EAI architecture allows another layer of process automation to exist over and above the processes encapsulated in existing applications.

Finally not only can the EAI technology help with integrating applications within the enterprise but it also provides strong support for applications across the entire value chain to be integrated. EAI technology is based upon open standards such as XML and HTTP, which allow the speed and cost of implementation to be greatly reduced. Features such as security, reliability, and scalability are often built-in.

\section{Strategic Advantages of Adopting Enterprise Application Integration Technology}

A well-integrated enterprise is in a much better position when faced with immense challenges of today and tomorrow. This section describes the strategic benefits of enterprise application integration.

Lower costs. A well-integrated enterprise can greatly reduce the amount of manual processing by automating processes such as data entry, error handling, and customer service. Automation of procurement by integrating order systems directly with suppliers can drive down the handling costs considerably. By providing improved visibility of supply and demand information across the value chain, an integrated enterprise can significantly reduce inventory costs.

\section{Figure 4 Integrating the Value Chain}




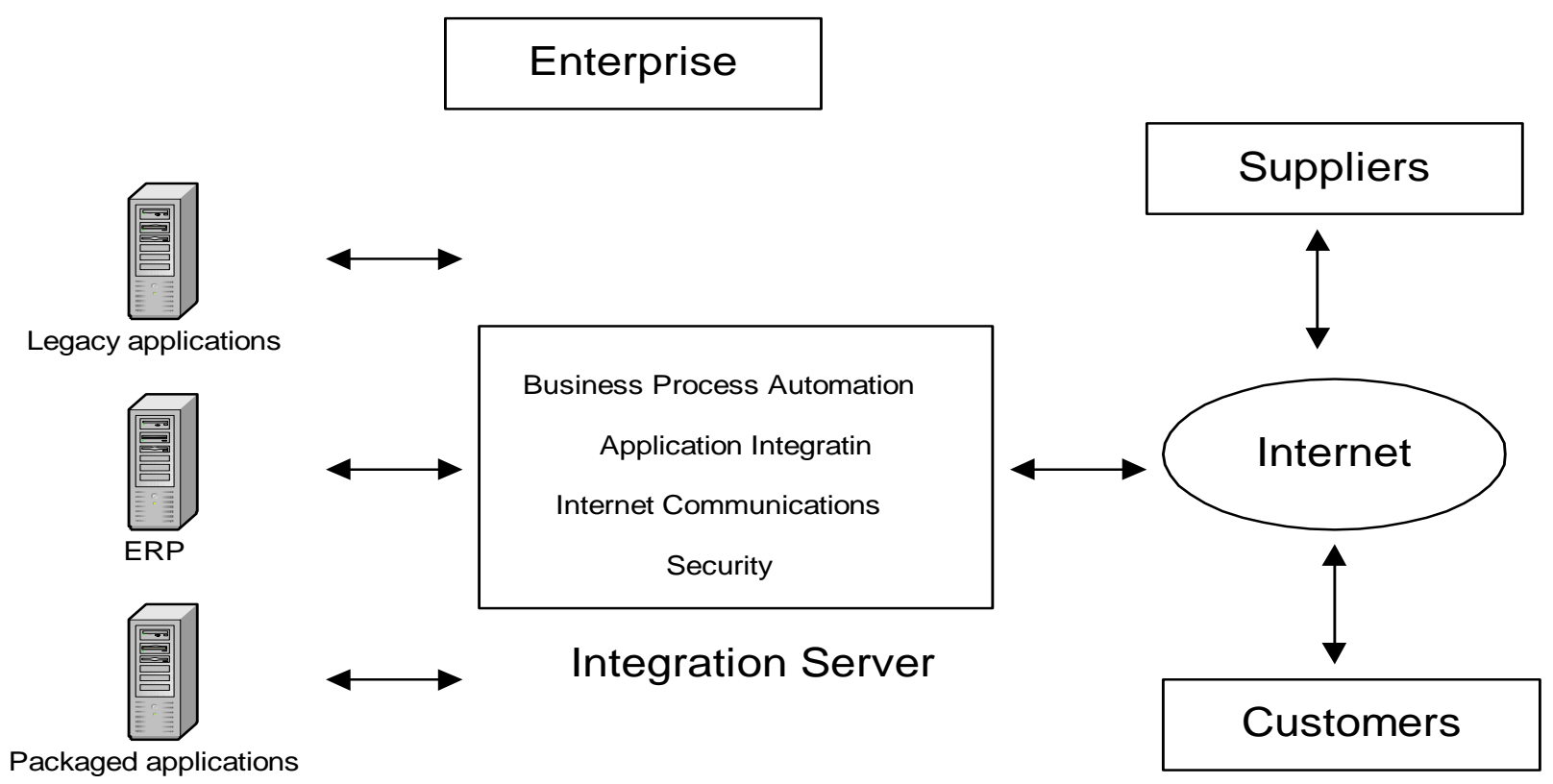

Tighter relationships along the value chain. An integrated architecture within the enterprise sets the stage for automating business processes that extend across the extended enterprise. An integrated architecture makes it easier to share information with partners. This can lead to improved quality by allowing partners to share information on product and service features, reliability, returns, and customer complaints. An integrated IT architecture is also a requirement for executing any E-business strategy. Inter-organizational data flows can be easily facilitated from such an architecture to enable inbound and outbound logistics. Operations can be tied more directly to the data flowing from the previously mentioned value chain activities, facilitating just-in-time production. These can all be done without proprietary/custom software development

Faster response to changes in the business environment. A well-integrated enterprise IT infrastructure provides the primary resources to enable an adaptive business infrastructure to face today's forever changing business environments. For example, rather than relying on summarized reports that are weeks or days old, a well-integrated set of systems can immediately inform the business if the marketing campaign is generating new demand or cannibalizing from existing demand. The customer service representative can easily find out exactly where in the pipeline a customer's order lies. The master scheduler can be immediately notified of the impact of a late purchase order or machine going down.

It is much easier to update and extend existing applications to support changed business processes. It is also easier to add additional IT resources, such as data warehouses and other new applications, to an existing IT architecture. These additions would not substantially affect the existing applications; it would only necessitate a modification of the integration server. This enables the architecture to assimilate state of the art technologies as they emerge in the environment.

Increased customer loyalty. Customers are increasingly demanding a single point of contact for all interactions with a business. The business needs to deliver full knowledge and responsibility for the transaction at this point of contact, even if the product or service was delivered through third parties. Integration of systems within and outside the boundaries of the enterprise allows it to deliver the quality of service required to retain loyal customers. An integrated business makes possible faster delivery of higher quality products at lower prices as a result of automated order processing, therefore eliminating the time-consuming manual steps typical in order fulfillment. Processing is speeded up throughout the entire value chain, from initial order placement, through product build, all the way to the 
final stage of shipping. The slow fax/email/voice methods for exchange of information, requiring multiple keyboarding of the same data, has become a thing of the past.

Automation of business processes. For example in a telecom company, an integrated and flow through process can facilitate customer representatives to take requests for service activation and configuring products based on provisioning and service availability information from the order management/provisioning applications and generate work orders in the in front-office applications, which are automatically propagated to the back-office for provisioning.

\section{Conclusions}

Success in today's business world requires an integrated enterprise IT infrastructure. Organizations must not only enable the seamless flow of information within the enterprise, but also between customers, supplies, and partners. The speed at which today's markets move requires that organizations move with greater haste in meeting customer expectations. Applications and resources must be made available regardless of corporate boundaries in a way that is flexible, scalable, reliable, and secure. However, today in many organizations applications are still poorly integrated. The traditional point-to-point approach to integration is inadequate as a solution to the integration challenges. ERP is only a partial and expensive solution. EAI enables the organization to achieve a less costly, more tightly integrated IT architecture. It also leverages existing applications and data to enable faster response to customer needs, ensuring greater customer loyalty. A truly strategic organization must have seamless inter-operability of its application architecture. Without EAI technology this would be impossible.

\section{References}

1. $\quad$ Aiken, P., Yoon, Y., Leong-Hong, B., "Requirements-Driven Data Engineering," Information \& Management, Vol. 35, Issue 3, March, 1999, pp. 155-168. (Problems with non-integrated systems at DOD)

2. Brakeley III, H.H., "The Promise of EAI,' Manufacturing Systems, March 2000, Vol. 18, Issue 3, p32p32.

3. Fulcher, J. "Make The Connection," Manufacturing Systems, March 2000, Vol. 18, Issue 3, pp. 76-81.

4. Hey, D. V., "Having It All," Personnel Management, Vol. 45, Issue 3, pp. 127-31, March 2000.

5. Leonard, B., Werner, C., "Examining Accounting and Integrated Web-Based Accounting Systems," Internal Auditing, Vol. 15, Mar/Apr 2000, pp. 14-19.

6. Lim, S. H., Juster, N., Pennington, A. D., "Enterprise Modeling and Integration: a Taxonomy of Seven Key Aspects," Computers in Industry, Vol. 34, 1997, pp. 339-359.

7. Linthicum, D. S., Enterprise Application Integration, Addison-Wesley, 2000.

8. $\quad$ McDougall, P., "IBM: Gunning for Growth,” Informationweek, May 14, 2001.

9. Soh, C., Kien, S.S., Tay-Yap, J., "Cultural Fits and Misfits: Is ERP A Universal Solution? Communications of the ACM," Vol. 43, No. 4, pp. 47-51, 2000.

10. Grimson, W. and Hasselbring, W., "The SI Challenge in Health Care," Communications of the ACM," Vol. 43, No. 6, pp. 48-56, June 2000.

11. Rowe, T., "Making the Business Case for Middleware," eAI Journal.

12. Yang, J. and Papazoglou, M. P., "Interoperation Support for Electronic Business," Communications of the ACM," Vol. 43, No. 6, pp. 39-47, June 2000. 